\title{
Indium-Tin-Oxide (ITO) as Stable and Effective Coating Material for Correlative Confocal and Immuno-Scanning Electron Microscopy Studies.
}

\author{
Andrea Falqui, ${ }^{1 \mathrm{a}}$ Simona Rodighiero, ${ }^{2}$ Elisa Sogne,,${ }^{2,1 \mathrm{a}}$ Bruno Torre, ${ }^{1 \mathrm{~b}}$ Roberta Ruffilli, ${ }^{3}$ Maura \\ Francolini, ${ }^{2,4}$ Cinzia Cagnoli, ${ }^{2}$ Enzo di Fabrizio ${ }^{1 \mathrm{~b}}$ \\ ${ }^{1}$ King Abdullah University for Science and Technology (KAUST), Thuwal 23955-6900, Kingdom of \\ Saudi Arabia: ${ }^{a}$ Biological and Environmental Sciences and Engineering Division; ${ }^{\mathrm{b}}$ Physical Sciences \\ and Engineering Division \\ ${ }^{2}$ Fondazione Filarete, Viale Ortles 22/4, 20139 Milano, Italy \\ ${ }^{3}$ CEMES/CNRS, 29 Rue Jeanne Marvig BP 94347, 31055 Toulouse Cedex 4, France \\ ${ }^{4}$ Università degli Studi di Milano, Via Vanvitelli 32, 20129 Milano, Italy
}

Correlating Confocal Microscopy (CM) and Electron Microscopy (EM) imaging of cells and tissues is a well-known method to understand the relations occurring between cellular structure and function. Conventional CM is capable to visualize the presence of either specific antigens by the use of immunofluorescent labeling or fluorescent proteins (FP), with resolution of few hundreds of nanometers. On the other hand, EM is capable to image the cellular ultrastructure down to nanometer scale. Putting together the information given by the two techniques on the same area of the specimen allows then to determine the antigen location on the cellular ultrastructure. EM imaging could be carried out on biological specimens both in transmission (TEM) and in scanning (SEM) mode. In both the EM approaches, to get information on antigen/protein distribution, cells can be labeled with antibodies conjugated with small $(<20 \mathrm{~nm})$ gold particles. In the case of SEM the secondary electrons (SE) are used to image the specimen surface morphology, whilst compositional contrast obtained by collecting backscattered electrons (BSE) allows to simultaneously localize the gold particles that labeled a cellular surface antigen.

A key point in the observation of the cellular ultrastructure is the preparation protocol followed. In particular, in the case of SEM imaging with surface immuno-labeling, the specimen has to be made electrically conductive, while preserving the compositional contrast needed to localize the gold nanoparticles acting as immuno-markers. This need brings to inevitably exclude heavy metals as coating agents, as they would completely mask the BSE signal coming from the nanogold immuno-markers.

Enough recent literature indicates two possible approaches to face such a problem. The first one consisted in using substrates covered by Indium Tin Oxide (ITO), being ITO a well known both optically transparent and stably conductive material [1]. In this approach the cells are not coated with any kind of conductive film since the ITO-covered substrates allow to not get specimen charging, but the overall quality of the cellular imaging so obtained is enough limited. Conversely, in the second approach the cells surface was coated with a very thin layer $(<5 \mathrm{~nm})$ of chromium, but with the main limitation due to the lack of conductivity because of chromium's fast oxidation that occurs if it is deposed under low vacuum condition or exposed to air [2].

We report here the correlative CM and immuno-SEM studies of HeLa cells and neurons grown on specific Ti-patterned glass substrates [3]. We studied if an optically transparent thin layer of ITO deposed by ion sputtering on the samples surface could be used in order to overcome the major restrictions resulting from the approaches reported in [1-2]. With this aim, we have first studied the optimal ITO layer thickness needed to obtain both good specimen conductivity and preservation of the BSE signal coming from gold nanoparticles used as immuno-markers. Second, we have estimated by 
Atomic Force Microscopy (AFM) the actual cells thickness range. That parameter was used to quantitatively determine by Monte Carlo simulations the most appropriate electron beam acceleration voltage to use for collecting BSE signal mainly coming from the immuno-labeled cells covered with the thinnest and conductive ITO coating layer. We have then found that such a coating film is stable over time, and capable to provide both suitable electrical conductivity, good SE production and preservation of the BSE signal coming from the gold immuno-markers. As a consequence, we show how it allows to easily perform correlative CM and immuno-SEM microscopy studies.

\section{References:}

[1] H. Pluk et al., J. Microsc. 233 (2009), p. 353.

[2] M. W. Goldberg, Methods Cell. Biol. 88 (2008), p. 109.

[3] L. Benedetti et al., Sci. Rep. 4 (2014), p. 7033.

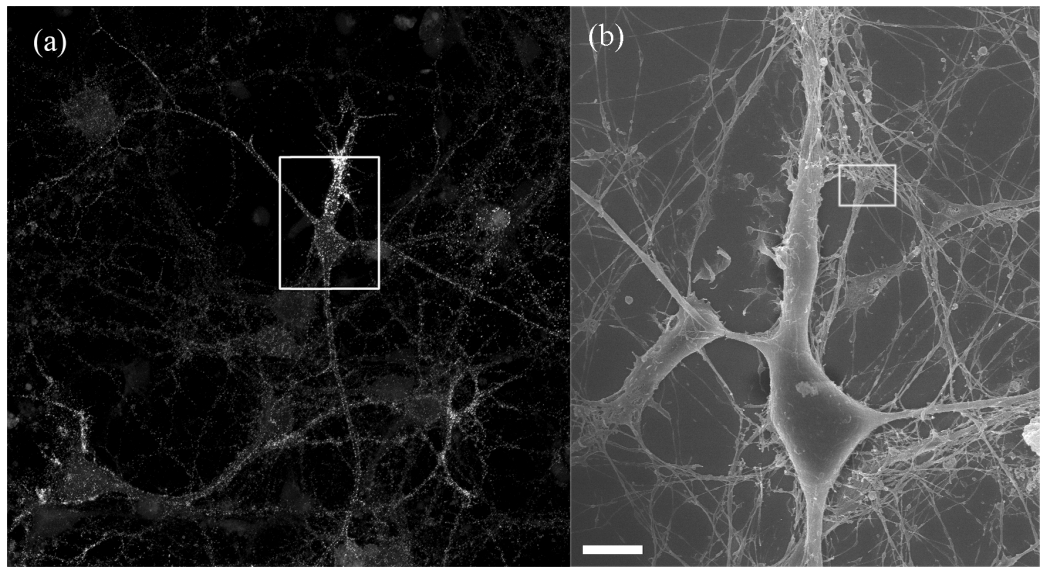

Figure 1. (a) Confocal Microscopy Images of primary cortical neurons where the subunit alfal of the $\mathrm{GABA}_{\mathrm{A}}$ receptor was tagged with ATTO 488 fuorophore. (b) SEM SE Low magnification image of the rectangular area reported in panel (a), after deposition of an ITO coating film of $20 \mathrm{~nm}$. Scale bar: $5 \mu \mathrm{m}$

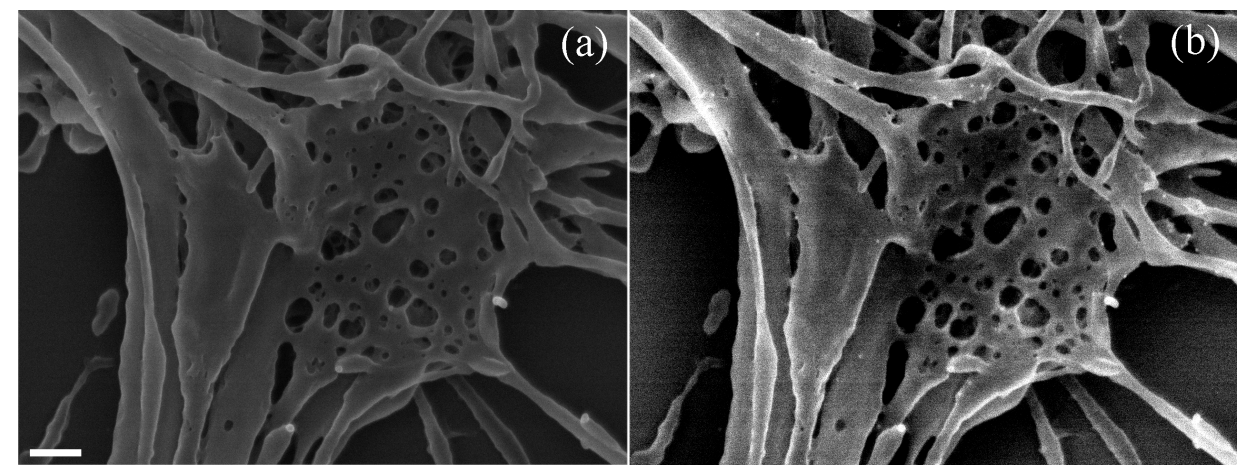

Figure 2. SEM imaging of primary cortical neurons where the subunit alfal of the GABA $\mathrm{A}$ was tagged with 12-nm nanogold markers and coated with an ITO film of $20 \mathrm{~nm}$ in thickness. (a) High resolution SE Images of the rectangular area reported in Figure 1 (b). (b): High resolution BSE image corresponding to panel (a): the gold immuno-markers can be easily observed. Scale bar: $50 \mathrm{~nm}$ 